\title{
AUTOMATED IMAGE MATCHING FOR 3-D POSE ESTIMATION OF KNEE USING SINGLE-PLANE FLUOROSCOPY
}

\author{
K. Kobayashi*, N. Tanaka**, M. Sakamoto*, Y. Tanabe*** \\ * Department of Health Sciences, Niigata University, Niigata, Japan \\ ** Graduate School of Science and Technology, Niigata University, Niigata, Japan \\ *** Department of Mechanical Engineering, Niigata University, Niigata, Japan
}

kobayasi@clg.niigata-u.ac.jp

\section{Introduction}

Total knee arthroplasty replaces a deformed joint with implants to restore the joint alignment and to facilitate the joint motion. Currently, the implant positioning is determined by X-ray and/or CT images of standing or lying posture, not accounting for knee kinematics under dynamic, weight-bearing conditions. This is due to the lack of knowledge of kinematics in physiological and pathological knees. Motion analysis systems using video cameras and skin-mounted markers have been used widely to study gross body motion. However these systems are less accurate for measuring motion of the underlying bone due to soft tissue motion artifact. The present study aims at developing a more direct method for measuring bone motion using fluoroscopy. Digitally reconstructed radiograph (DRR) created using a 3D bone model derived from CT data was projected to the fluoroscopic image of knee. Gradient-based image matching was performed to estimate the 6 degree-of-freedom (DOF) parameters representing the $3 \mathrm{D}$ relative pose of femur and tibia. Estimated pose parameters were compared with true values measured using a $3 \mathrm{D}$ coordinated measuring machine.

\section{Materials and Methods}

True value of relative pose: The study was carried out on three fresh porcine cadaver knees. Three sphere markers were bonded to each femur and tibia. The femur and tibia were fixed using an external fixation device, resembling an anatomical position. The central coordinates of the sphere markers were measured by a $3 \mathrm{D}$ coordinate measuring machine (BH504, MITUTOYO, Japan) to define the absolute position of each bone. The relative pose between the two bones was derived from their absolute positions, and used as the true value.

DRR creation using bone volume model: Three dimensional bone volume models were generated by bone segmentation of CT scan data of femur and tibia (field of view: $640 \times 512$ pixel, pixel size: $0.35 \times 0.35 \mathrm{~mm}^{2}$, slice thickness: $1 \mathrm{~mm}$ ). The original anisotropic voxel, $0.35 \times 0.35 \times 1.0 \mathrm{~mm}^{3}$, was transformed into an isotropic voxel with $1.0 \times 1.0 \times 1.0 \mathrm{~mm}^{3}$ to improve the quality of DRR. DRR was created by projecting the all voxels to the image plane by the following equation.

$$
I(u, v)=I_{0} e^{-\sum_{i=1}^{n} \alpha \mu_{i}}
$$

Where $I_{0}$ indicates the dynamic range, $n$ the number of voxels projected to a point $(u, v)$ on the image plane, $\alpha$ the attenuation factor, $\mu_{i}$ the voxel value. Values of $I_{0}$ and $\alpha$ were selected so that the value of $I$ matched with that of the actual fluoroscopic image.

Automated pose estimation algorithm: To estimate the 6 DOF parameters of bone, a gradient difference of fluoroscopic image and DRR [1] was used as a similarity measure. Maximizing the similarity measure between the 2 images was carried out using a downhill simplex algorithm [2]. To compare the relative pose of femoral model with respect to tibial model, 6 DOF parameters of tibial model was first determined with a manual image matching [3]. Then the femoral model was placed according to the true value of relative pose. Finally, the automated image matching algorithm was performed on the femoral model. The estimated relative pose with respect to the tibial model was compared with the true value of relative pose. Ten single plane fluoroscopic images with different flexion angles and different directions of image acquisition were studied.

\section{Results and Discussion}

Results of the absolute errors in estimating 6 DOF parameters are listed in Table 1. Errors in two out-of-plane (y and z) rotations, $2.8 \mathrm{deg}$ and $1.7 \mathrm{deg}$, and one out-of-plane (x) translation, $3.9 \mathrm{~mm}$ are larger than those in in-plane $(\mathrm{x})$ rotation, $0.7 \mathrm{deg}$, and two in-plane (y and $\mathrm{z}$ ) translations, $0.5 \mathrm{~mm}$ and $0.7 \mathrm{~mm}$. These accuracies are comparable to previous report [4]. Ideally, errors could be negligible since the automated image matching started from the true value. However, several factors such as less clear bone edge due to surrounding soft tissue and imperfect bone volume models would make the accuracy worse. Therefore, although the present result shows a potential of the proposed method for the accurate analysis of in vivo knee kinematics, further research is required to identify sources of errors responsible for inaccuracy.

Table 1 Mean (SD) of absolute errors in estimating rotation and translation parameters $(n=10)$

\begin{tabular}{cccccc}
\hline \multicolumn{3}{c}{ Rotation } & \multicolumn{3}{c}{ Translation } \\
\hline $\mathrm{x}, \mathrm{deg}$ & $\mathrm{y}, \mathrm{deg}$ & $\mathrm{z}, \mathrm{deg}$ & $\mathrm{x}, \mathrm{mm}$ & $\mathrm{y}, \mathrm{mm}$ & $\mathrm{z}, \mathrm{mm}$ \\
\hline 0.7 & 2.8 & 1.7 & 3.9 & 0.5 & 0.7 \\
$(0.4)$ & $(2.3)$ & $(1.8)$ & $(2.5)$ & $(0.6)$ & $(0.6)$ \\
\hline
\end{tabular}

\section{Conclusions}

A method for analyzing in vivo knee kinematics using single plane fluoroscopy and $3 \mathrm{D}$ bone models was developed. Digitally reconstructed radiographs (DRRs) were generated from $3 \mathrm{D}$ bone volume models by a voxel projection technique. The relative $3 \mathrm{D}$-pose (full 6 DOF parameters) between porcine femur and tibia were determined by matching the DRR of each bone model with the fluoroscopic image by maximizing the similarity measure between the 2 images. The true value of the relative pose was measured by a $3 \mathrm{D}$ coordinate measuring machine. The mean errors of the two out-of-plane rotation parameters were $2.8 \mathrm{deg}$ and $1.7 \mathrm{deg}$, and the in-plane parameter was $0.7 \mathrm{deg}$. The error of out-of-plane translation parameter was $3.9 \mathrm{~mm}$ while the two out-of-plane parameters were $0.5 \mathrm{~mm}$ and $0.7 \mathrm{~mm}$.

\section{References}

[1] Penny GP, Weese J, Little JA, Desmedt P, Hill DLG, Hawkes DJ: A comparison of similarity measures for use in 2-D-3-D medical image registration, IEEE Trans Med Imging, 17-4: 586-95, 1998.

[2] Nelder JA, Mead R: A simplex method for function minimization, Comp J, 7: 308-13, 1965.

[3] Kobayashi K, Tanaka N, Sakamoto M, Tanabe Y: Estimation of 3Dpose of knee using single plane fluoroscopy and 3D bone model, Proc Int Conf Advance Tech Exp Mech, Fukuoka, Japan, 2007 (in press).

[4] Fregly BJ, Rahman HA, Banks SA: Theoretical accuracy of modelbased shape matching for measuring natural knee kinematics with single-plane fluoroscopy, $J$ Biomech Eng, 127: 692-99, 2005. 